\title{
Demonstration of a tilted-pulse-front pumped plane-parallel slab terahertz source
}

\author{
Priyo Syamsul Nugraha, ${ }^{1,2}$ Gergö Krizsán, ${ }^{1}$ Csaba Lombosi, ${ }^{1}$ László Pálfalvi, ${ }^{3}$ György Tóth, ${ }^{3}$ \\ Gábor AlmásI, ${ }^{3}$ József András FülöP, ${ }^{1,2,4, *}$ (1) AND János Hebling ${ }^{1,2,3}$ \\ ${ }^{1}$ Szentágothai Research Centre, University of Pécs, 7624 Pécs, Hungary \\ ${ }^{2}$ MTA-PTE High-Field Terahertz Research Group, 7624 Pécs, Hungary \\ ${ }^{3}$ Institute of Physics, University of Pécs, 7624 Pécs, Hungary \\ ${ }^{4}$ ELI-ALPS, ELI-Hu Nonprofit Ltd., 6720 Szeged, Hungary \\ ${ }^{*}$ Corresponding author: fulop@fizika.ttk.pte.hu
}

Received 23 November 2018; revised 7 January 2019; accepted 12 January 2019; posted 14 January 2019 (Doc. ID 351457); published 14 February 2019

\begin{abstract}
A new type of tilted-pulse-front pumped terahertz $(\mathrm{THz})$ source has been demonstrated, which is based on a $\mathrm{LiNbO}_{3}$ plane-parallel slab with an echelon structure on its input surface. Single-cycle pulses of $1 \mu \mathrm{J}$ energy and $0.30 \mathrm{THz}$ central frequency have been generated with $5 \times 10^{-4}$ efficiency from such a source. One order-of-magnitude increase in efficiency is expected by pumping a cryogenically cooled echelon of increased size and thickness with a Ti:sapphire laser. The use of a plane-parallel nonlinear optical crystal slab enables straightforward scaling to high $\mathrm{THz}$ pulse energies and the production of a symmetric THz beam with a uniform pulse shape for good focusability and high field strength. () 2019 Optical Society of America
\end{abstract}

https://doi.org/10.1364/OL.44.001023

Provided under the terms of the OSA Open Access Publishing Agreement

The study and control of materials with extremely strong terahertz $(\mathrm{THz})$ fields $[1,2]$, or the acceleration and manipulation of electrons [3] and protons [4], are emerging applications which require $\mathrm{THz}$ sources with unprecedented parameters. Besides the pulse energy, an excellent focusability is essential to achieve the highest possible field strengths.

Optical rectification of ultrashort laser pulses with a tilted pulse front in lithium niobate (LN) [5] has become a standard technique for efficient $\mathrm{THz}$ generation. Conventionally, a prism-shaped LN crystal is used with a large wedge angle equal to the pulse-front tilt $\left(63^{\circ}\right)$. Such a source geometry results in a nonuniform pump propagation length across the beam, which can lead to a spatially varying interaction length for $\mathrm{THz}$ generation [6]. This negatively affects the $\mathrm{THz}$ beam quality and, consequently, the focusability, thereby limiting the achievable field strength. Lateral beam (and eventually waveform) nonuniformity is especially problematic in high-energy $\mathrm{THz}$ sources [7], where a large-diameter pump beam is needed.

Different approaches have been proposed to mitigate the limitations of tilted-pulse-front pumped $\mathrm{THz}$ sources. The contact-grating technique has been proposed to eliminate imaging errors present in conventional setups utilizing a grating and an imaging lens or telescope [8]. While a plane-parallel contact-grating $\mathrm{THz}$ source with uniform interaction length could be successfully demonstrated in a $\mathrm{ZnTe}$ semiconductor [9], the realization of a practical source in LN turned out to be very challenging $[10,11]$. The technical requirements on the contact grating can be somewhat relaxed by combining a conventional external pulse-front-tilting setup with a contact grating [12]. However, even in such hybrid setups, a prism-shaped LN crystal needs to be used with a $\sim 30^{\circ}$ wedge angle. This is still sufficiently large to cause strong transversal nonuniformity across centimeter-sized beams.

Recently, we have proposed a modified hybrid approach to provide a uniform interaction length across a large pump and $\mathrm{THz}$ beams [13]. In this setup, the nonlinear medium is a plane-parallel LN slab with an echelon structure on its input surface. Inside the LN slab, a segmented tilted pulse front is formed with an average tilt angle as required by phase matching. We note that a reflective echelon has been used earlier for tilting the average pump pulse front for efficient $\mathrm{THz}$ generation in LN, but this approach still requires the use of a prism-shaped LN crystal with the same wedge angle as in the conventional setup $[14,15]$.

In this Letter, we demonstrate the hybrid tilted-pulse-front pumped $\mathrm{THz}$ source which utilizes a plane-parallel LN slab with an echelon structure. This new technology opens up the way for constructing efficient sources of high-energy $\mathrm{THz}$ pulses with excellent focusability for high field strengths.

Figure 1 illustrates the importance of a uniform interaction length across the pump and $\mathrm{THz}$ beams. While the output $\mathrm{THz}$ waveform strongly varies across the beam in a conventional prism-shaped LN crystal [Fig. 1(a)], the waveform can be nearly perfectly uniform in a plane-parallel LN slab with an echelon [Fig. 1(b)]. This feature is essential for a straightforward scalability to extremely high $\mathrm{THz}$ pulse energies by simply increasing the pump spot size and energy, as well as for producing the highest possible $\mathrm{THz}$ peak field strengths by the excellent focusability. 


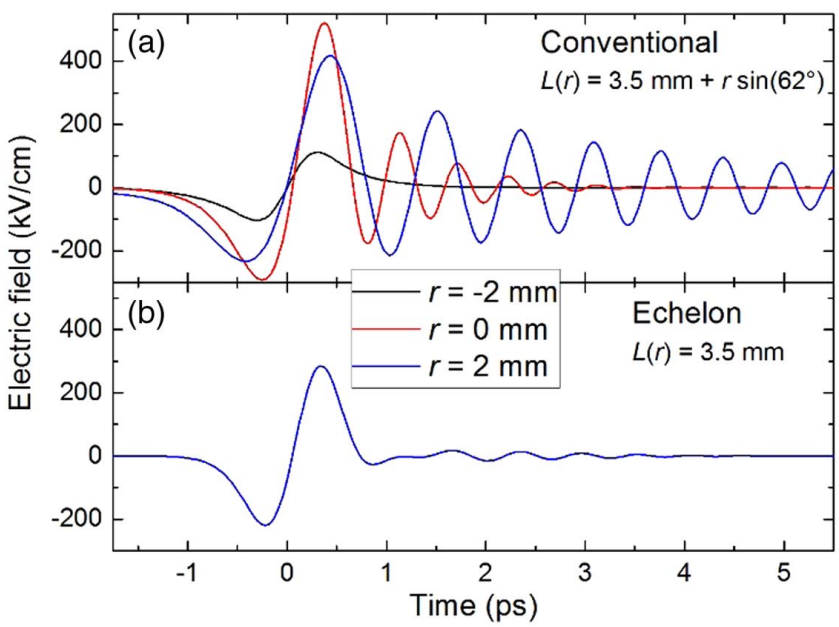

Fig. 1. Calculated $\mathrm{THz}$ pulse waveforms at different positions across the $\mathrm{THz}$ beam for a LN prism (a) and a plane-parallel LN slab (b). The lateral position in the input pump beam is labeled by $r$, whereby $r=0$ corresponds to the beam center. $L(r)$ is the corresponding material length along the $\mathrm{THz}$ propagation direction.

The investigated hybrid-type setup [13] is a combination of the conventional scheme, containing a diffraction grating and imaging optics, and a nonlinear material with an echelon profile on its entrance surface [nonlinear echelon slab, NLES, Fig. 2(a)]. Pump pulses of $200 \mathrm{fs}$ pulse duration and $1030 \mathrm{~nm}$ central wavelength were delivered by a cryogenically cooled $\mathrm{Yb}: \mathrm{CaF}_{2}$ regenerative amplifier operating at $1 \mathrm{kHz}$ repetition rate. Up to about $2.5 \mathrm{~mJ}$ pump pulse energy was used in the experiment. At the pump wavelength used and at room temperature, a pulse-front tilt of about $63^{\circ}$ is required for phase matching in LN. In the case of the NLES, this pulse-front tilt angle needs to be produced in air at the entrance of the crystal. This is in contrast to the conventional setup where a larger angle of $77^{\circ}$ is needed in air, which is then reduced according to $\tan \left(\gamma_{\mathrm{LN}}\right)=\tan \left(\gamma_{\text {air }}\right) / n_{\mathrm{g}}$ to $63^{\circ}$ by entering into the $\mathrm{LN}$ prism $[16,17]$. Here $n_{\mathrm{g}}=2.215$ is the group index of $\mathrm{LN}$ at the $1030 \mathrm{~nm}$ pump wavelength. The smaller tilt angle is advantageous for reducing imaging errors of the grating-lens system [6].

When the pump beam with the pulse-front tilt angle of $\gamma_{\text {air }}=63^{\circ}$ in air enters the NLES, the local tilt angle is reduced to $\gamma_{\mathrm{LN}}=42^{\circ}[$ Fig. $2(\mathrm{a})]$, according to $\tan \left(\gamma_{\mathrm{LN}}\right)=\tan \left(\gamma_{\mathrm{air}}\right) / n_{\mathrm{g}}$.
However, the average pulse-front tilt angle across the pump spot remains unchanged. This leads to the formation of a segmented pulse front [13].

A transmission grating with $1600 \mathrm{~mm}^{-1}$ line density (LightSmyth Technologies, T-1600-1030S) was used at an incidence angle of $55.5^{\circ}$, close to the Littrow angle. To ensure the necessary pulse-front tilt at the crystal, the imaging lens (a near-infrared achromat with $25 \mathrm{~cm}$ focal length) had to provide a magnification of about 1.4 times. We note that a more optimal design would involve demagnification, rather than magnification, as then the pump intensity would be higher at the crystal than at the grating. However, we had no appropriate grating to build such a setup. Grating damage, indeed, was limiting the useful pump intensity in our case. The pump spot size at the NLES, perpendicular to the pump propagation direction, was $5.5 \mathrm{~mm} \times 5.1 \mathrm{~mm}$ (horizontal $\times$ vertical, at $1 / e^{2}$ of the peak intensity).

A prototype LN NLES device has been manufactured by diamond milling (Kugler GmbH., Salem, Germany). The dimensions of the slab were $5 \mathrm{~mm} \times 8 \mathrm{~mm}$ along and perpendicular to the steps, respectively. The slab thickness was $L=3 \mathrm{~mm}$. The width of the echelon steps, through which the pump beam entered the slab, was $w=50 \mu \mathrm{m}$ [Fig. 2(a)]. The height of the steps, along the pump propagation direction, was $h=92 \mu \mathrm{m}$. Such step geometry is consistent with a pulsefront tilt angle of about $62^{\circ}$, required for velocity matching at a cryogenic temperature $(100 \mathrm{~K})$, rather than at room temperature. For the sake of simplicity, in this proof-of-principle experiment, the NLES was used at room temperature.

The optic axis ( $Z$-axis) of the LN slab was parallel to the (vertical) echelon lines. The slab was $Y$-cut. The polarization of the pump beam was perpendicular to the plane of Fig. 2(a). The generated $\mathrm{THz}$ beam had the same polarization direction. The $\mathrm{THz}$ pulse energy has been measured by a calibrated pyroelectric detector (THZ 20, Sensor- und Lasertechnik). THz waveforms have been measured by electro-optic sampling in a [110]-cut $1 \mathrm{~mm}$ thick GaP using conventional ellipsometric balanced detection. A small fraction of the $200 \mathrm{fs}$ pump pulses was used for the sampling.

Perspective-view optical microscope images of the echelon structure are shown in Fig. 3. The surface flatness along the entrance step faces of width $w$ were estimated from interferometric measurements to be about $\lambda / 10$ peak-to-peak at the pump wavelength used here, and the rms roughness was $25 \mathrm{~nm}$. It could also be deduced from microscope inspection
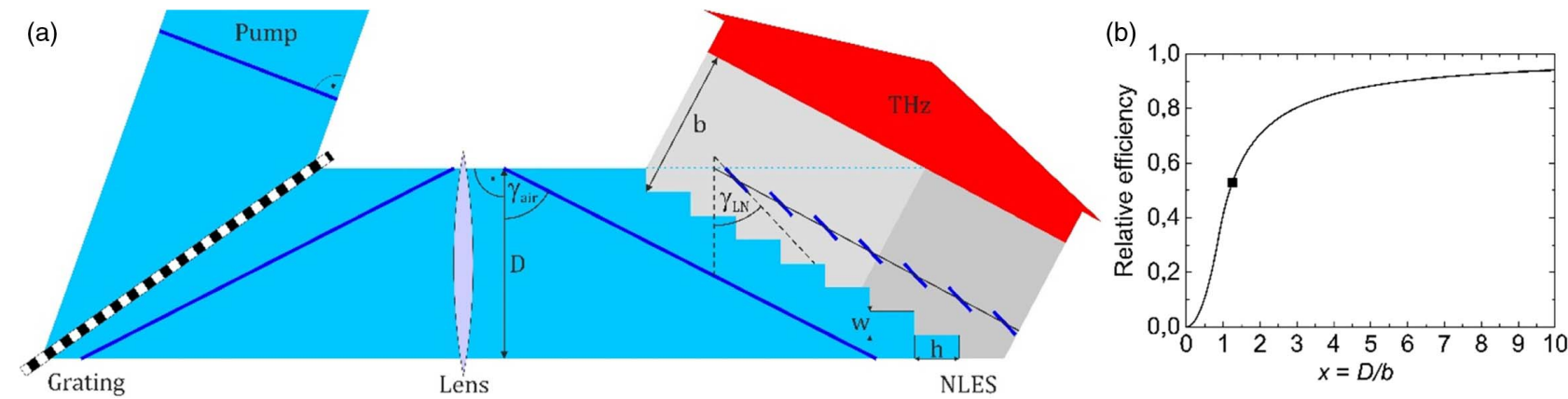

Fig. 2. (a) Scheme of the experimental setup. The pump pulse fronts are indicated at different positions in the setup. The angular dispersion of the pump is not shown. Inside the NLES, the pump pulse front is segmented. (b) Relative THz generation efficiency owing to a reduced interaction length in the light gray area in (a) as a function of the relative pump beam size. 

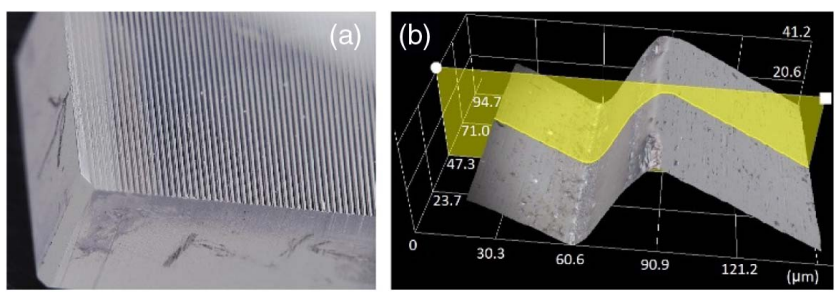

Fig. 3. (a) Perspective view of the prototype echelon slab structure taken at 20x magnification. (b) Reconstructed surface topology model of a single step of the echelon structure (700x magnification). The images were recorded by a Hirox RH-2000 digital microscope by Emilien Leonhardt from Hirox Europe.

that the step edges of the echelon profile were rounded [see Fig. 3(b)]. This resulted in an estimated reduction of the effective area by about $20 \%$ to $30 \%$.

Figure 4 shows the measured THz pulse energy (left axis) and the pump-to-THz energy conversion efficiency (right axis) as functions of the pump pulse energy (bottom axis) and the pump intensity (top axis). THz pulses with up to $1.0 \mu \mathrm{J}$ energy have been generated with $1.9 \mathrm{~mJ}$ of pump reaching the input side of the NLES. The efficiency was $5.1 \times 10^{-4}$. A nearly quadratic increase of the $\mathrm{THz}$ energy and a corresponding linear increase of the efficiency can be observed up to about $1.0 \mathrm{~mJ}$ pump energy ( $25 \mathrm{GW} / \mathrm{cm}^{2}$ intensity). At higher pump energy (intensity) the saturation of the efficiency is observed. The useful pump energy (intensity) and, consequently, the $\mathrm{THz}$ energy, has been limited by the damage of the pulsefront-tilting grating, as mentioned above.

For comparison, the obtained $\mathrm{THz}$ generation efficiency was smaller by a factor of three for the NLES setup than for a conventional tilted-pulse-front pumped setup consisting of a reflection grating and an achromat lens, used for producing a $77^{\circ}$ tilt angle of the pump intensity front before the prismshaped LN crystal with a wedge angle of $63^{\circ}$. According to a simple estimation, however, from this factor of three the small size of the NLES is responsible for a factor of nearly two. For a small value of $x=D / b$ [Fig. 2(a)], the effective generation length will be shorter than the crystal thickness on a large part of the crystal, indicated by the light gray area inside the NLES

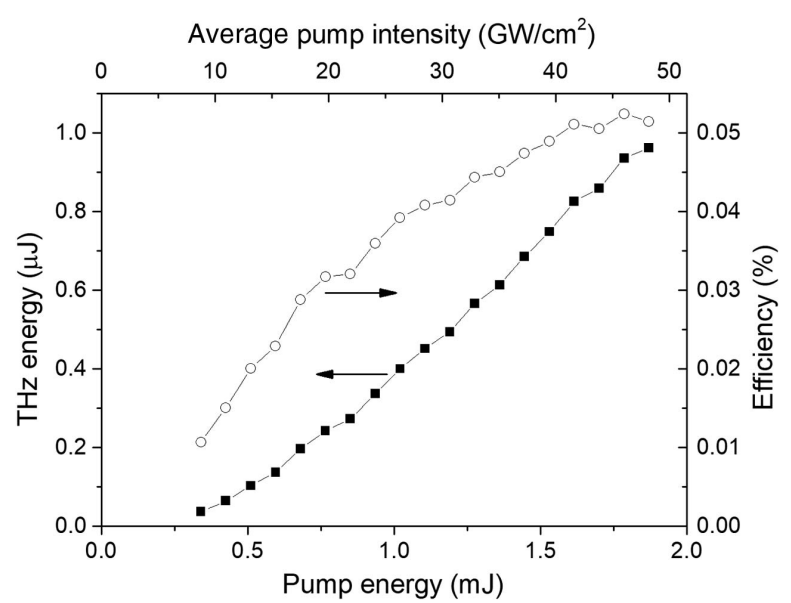

Fig. 4. Measured $\mathrm{THz}$ pulse energy and $\mathrm{THz}$ generation efficiency as functions of the pump energy and intensity. in Fig. 2(a). This causes a relative decrease in the $\mathrm{THz}$ generation efficiency by a factor $\eta_{\text {rel }}=x^{2} /\left(3 \sin ^{2} \gamma\right)$ for $x<\sin \gamma$ and $\eta_{\mathrm{rel}}=1-2 \sin \gamma /(3 x)$ for $x \geq \sin \gamma$ [Fig. 2(b)]. In this proof-of-principle experiment, from the $8 \mathrm{~mm}$ full width of the NLES crystal, the THz generation length was equal to the $3 \mathrm{~mm}$ crystal thickness only along a $2.4 \mathrm{~mm}$ broad part $(x \approx 1.2)$, leading to a relative efficiency of $\eta_{\text {rel }}=52 \%$ [indicated by the symbol in Fig. 2(b)] in comparison to the ideal case of $x \gg 1$. This problem can be eliminated by using crystals and pump beams with much larger transversal sizes than the thickness of the NLES. In addition, the rounded part of the echelon steps at the edges [Fig. 3(b)] could also have caused some efficiency decrease of the NLES setup, as compared to the ideal case.

By using a larger NLES of better quality, the efficiency can be expectedly improved in several ways. (1) Cryogenic cooling of the NLES reduces the absorption in the $\mathrm{THz}$ range and can result in a $2.5 \times$ to $3.0 \times$ increase of the efficiency $[7,18,19]$. (2) As discussed above, using a significantly larger NLES can result in a $2 \times$ increase of the efficiency. (3) A larger crystal length $L$ ( $5 \mathrm{~mm}$ instead of $3 \mathrm{~mm}$ ), with a sufficiently large aperture, can lead to further enhancement of the efficiency by a factor of $1.3 \times$ [18]. (4) Because of the smaller diffraction of the segmented pump beam for a shorter wavelength of $800 \mathrm{~nm}$, using a Ti:sapphire laser instead of an $\mathrm{Yb}$-based one can result in a further $40 \%$ increase of the efficiency [18]. Taken together, improvements (1)-(4) with a NLES of better quality can increase the efficiency by about one order of magnitude, reaching $\eta=0.5 \%$.

Examples of $\mathrm{THz}$ waveforms measured at different pump energies are shown in Fig. 5. These measurements have been carried out with a slightly modified setup, where a $250 \mathrm{~cm}$ focal length cylindrical lens has been used for imaging, in combination with a 2-m focal length cylindrical lens. This was used for the reduction of the vertical beam size at the NLES for enabling higher pump intensity without the risk of grating damage. The measured waveforms are nearly single-cycle, in reasonably good accordance with the result of numerical simulation, also shown in Fig. 5. The absence of significant ringing on the trailing part can be an important advantage when these pulses are used in particle acceleration setups [20]. The intensity spectra

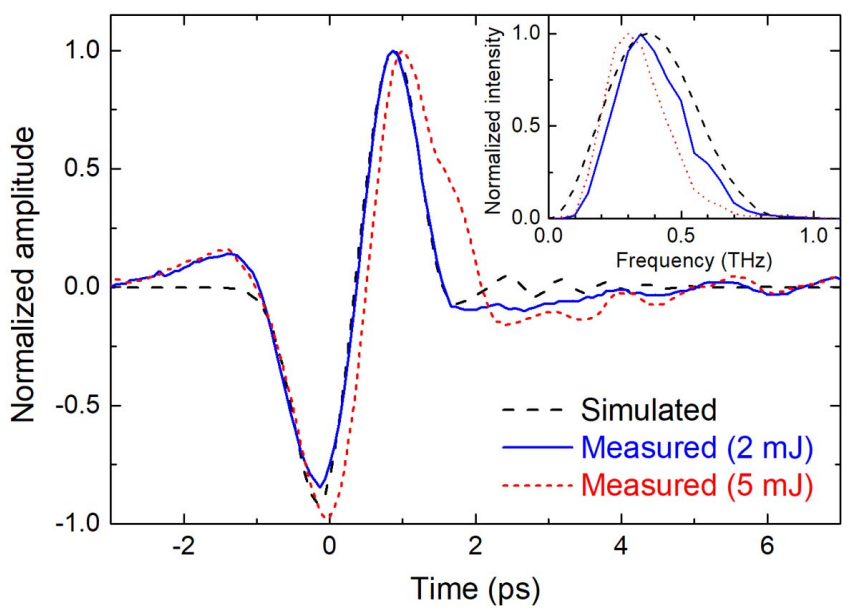

Fig. 5. Measured (at two different pump pulse energies) and simulated $\mathrm{THz}$ pulse waveforms and their intensity spectra (inset). 
calculated from the measured and simulated waveforms can be seen in the inset of Fig. 5. The simulated spectrum reaches its maximum at $0.38 \mathrm{THz}$. The spectral peaks of the measured $\mathrm{THz}$ pulses are at somewhat lower frequencies. The $0.3-$ $0.4 \mathrm{THz}$ mean frequency is suitable for particle acceleration applications.

Increasing the pump energy from 2 to $5 \mathrm{~mJ}$ results in the appearance of a clear shoulder on the trailing part of the waveform and a shift of the spectral intensity maximum from 0.35 to $0.30 \mathrm{THz}$. Besides these changes, the saturation of the efficiency can be observed. These observations are clear indications of nonlinear effects. Previously, nonlinear effects such as self-phase modulation, cascading effect, combination of group-velocity dispersion and angular dispersion, and stimulated Raman scattering were simulated in conventional tilted-pulse-front pumped setups [21,22]. Another possible nonlinear effect is shift current generation by the $\mathrm{THz}$ pulse, which was investigated in LN crystal, but not in the $\mathrm{LN} \mathrm{THz}$ source [23]. Since the numerical model used in this Letter does not take into account these nonlinear effects, it cannot explain the observed efficiency saturation, as well as the waveform and spectral distortions. However, we would like to emphasize that the investigation of the nonlinear effects is less complicated for a plane-parallel crystal than for a prism-shaped LN crystal of $63^{\circ}$ wedge angle, as it was the case in the previous investigations.

In conclusion, a new type of tilted-pulse-front pumped $\mathrm{THz}$ source has been demonstrated, which is based on a $\mathrm{LiNbO}_{3}$ plane-parallel slab with an echelon structure on its input surface. Single-cycle pulses of $1.0 \mu \mathrm{J}$ energy and $0.30 \mathrm{THz}$ central frequency have been generated with $5.1 \times 10^{-4}$ efficiency from such a source. One order-of-magnitude increase in efficiency is expected by pumping a cryogenically cooled echelon of increased size and thickness with a Ti:sapphire laser. The use of a plane-parallel nonlinear optical crystal slab enables straightforward scaling to high $\mathrm{THz}$ pulse energies. The pump pulse duration, and the $\mathrm{THz}$ absorption and dispersion, are uniform across the $\mathrm{THz}$ beam profile. This advantage, together with the reduced pump beam imaging errors [13], enables us to produce a symmetric $\mathrm{THz}$ beam with a uniform pulse shape for excellent focusability and high field strength. Such a source can be especially suitable to generate low $\mathrm{THz}$ frequencies for $\mathrm{THz}$-driven particle acceleration and other applications.

Finally, it is useful to notice that, because of the reduced necessary tilt angle and corresponding angular dispersion of the pre-tilt generation setup, a single transmission grating [instead of a grating-lens combination; Fig. 2(a)] could be used in front of the nonlinear echelon slab. Our preliminary experiment with such a simplified, but not an optimized configuration, resulted in $10^{-4}$ efficiency at about $13.6 \mathrm{GW} / \mathrm{cm}^{2}$ pump intensity, which is only about two times smaller than that with imaging (Fig. 4).
Funding. National Research, Development and Innovation Office (NKFIH) (125808); European Social Fund (ESF) (EFOP-3.6.2-16-2017-00005).

Acknowledgment. The authors thank Aladár Czitrovszky and Attila Tibor Nagy from the Wigner Research Centre for Physics (Budapest, Hungary) for the help in surface characterization of the NLES sample using a Zygo 7100 optical surface profiler. They also thank the Grimas Kft. for granting access to the Hirox RH-2000 digital microscope.

\section{REFERENCES AND NOTES}

1. T. Kampfrath, K. Tanaka, and K. A. Nelson, Nat. Photonics 7, 680 (2013).

2. D. Nicoletti and A. Cavalleri, Adv. Opt. Photonics 8, 401 (2016).

3. D.-F. Zhang, A. Fallahi, M. Hemmer, X.-J. Wu, M. Fakhari, Y. Hua, H Cankaya, A.-L. Calendron, L. E. Zapata, N. H. Matlis, and F. X. Kaertner, Nat. Photonics 12, 336 (2018).

4. L. Pálfalvi, J. A. Fülöp, G. Tóth, and J. Hebling, Phys. Rev. ST Accel. Beams. 17, 031301 (2014).

5. J. Hebling, G. Almási, I. Z. Kozma, and J. Kuhl, Opt. Express 10, 1161 (2002).

6. J. A. Fülöp, L. Pálfalvi, G. Almási, and J. Hebling, Opt. Express 18, 12311 (2010).

7. J. A. Fülöp, Z. Ollmann, C. Lombosi, C. Skrobol, S. Klingebiel, L. Pálfalvi, F. Krausz, S. Karsch, and J. Hebling, Opt. Express 22 20155 (2014).

8. L. Pálfalvi, J. A. Fülöp, G. Almási, and J. Hebling, Appl. Phys. Lett. 92 171107 (2008).

9. J. A. Fülöp, G. Polónyi, B. Monoszlai, G. Andriukaitis, T. Balciunas, A. Pugzlys, G. Arthur, A. Baltuska, and J. Hebling, Optica 3, 1075 (2016).

10. M. Tsubouchi, K. Nagashima, F. Yoshida, Y. Ochi, and M. Maruyama, Opt. Lett. 39, 5439 (2014).

11. Z. Ollmann, J. Hebling, and G. Almási, Appl. Phys. B 108, 821 (2012).

12. L. Pálfalvi, Z. Ollmann, L. Tokodi, and J. Hebling, Opt. Express 24, 8156 (2016)

13. L. Pálfalvi, G. Tóth, L. Tokodi, Z. Márton, J. A. Fülöp, G. Almási, and J. Hebling, Opt. Express 25, 29560 (2017).

14. B. K. Ofori-Okai, P. Sivarajah, W. Ronny Huang, and K. A. Nelson, Opt. Express 24, 5057 (2016).

15. K. Murate, M. J. Roshtkhari, X. Ropagnol, and F. Blanchard, Opt. Lett. 43, 2090 (2018)

16. J. Hebling, Opt. Quantum Electron. 28, 1759 (1996).

17. J. A. Fülöp and J. Hebling, Recent Optical and Photonic Technologies, K. Y. Kim, ed. (InTech, 2010), Chap. 11

18. According to numerical simulations using an improved version of the model described in Ref. [13]. The details of the model will be published separately.

19. S.-W. Huang, E. Granados, W. R. Huang, K.-H. Hong, L. E. Zapata, and F. X. Kärtner, Opt. Lett. 38, 796 (2013)

20. Z. Tibai, M. Unferdorben, S. Z. Turnár, A. Sharma, J. A. Fülöp, G. Almási, and J. Hebling, J. Phys. B 51, 134004 (2018).

21. K. Ravi, W. R. Huang, S. Carbajo, X.-J. Wu, and F. X. Kaertner, Opt. Express 22, 20239 (2014).

22. K. Ravi, W. R. Huang, S. Carbajo, E. A. Nanni, D. N. Schimpf, E. P. Ippen, and F. X. Kaertner, Opt. Express 23, 5253 (2015).

23. C. Somma, K. Reimann, C. Flytzanis, T. Elsaesser, and M. Woerner, Phys. Rev. Lett. 112, 146602 (2014). 